\title{
L'avènement des peines alternatives en droit pénal camerounais : contours et concours de l'une des innovations de la réforme législative du 12 juillet 2016
}

The advent of alternative sentences in Cameroon, penal law: concurring contours of one of the novelties in the legislative reform dated July 12, 2016

Dieudonné Soweng

\section{CpenEdition}

\section{Journals}

Édition électronique

URL : http://journals.openedition.org/add/1656

DOI : 10.4000/add. 1656

ISSN : 2606-1988

\section{Éditeur}

Presses universitaires de Rouen et du Havre

\section{Édition imprimée}

Date de publication : 1 mai 2019

Pagination : 185-213

ISBN : 979-10-240-1293-3

ISSN : 1955-0855

\section{Référence électronique}

Dieudonné Soweng, «L'avènement des peines alternatives en droit pénal camerounais : contours et concours de l'une des innovations de la réforme législative du 12 juillet 2016 », Les Annales de droit [En ligne], 13 | 2019, mis en ligne le 09 décembre 2019, consulté le 24 janvier 2021. URL : http:// journals.openedition.org/add/1656 ; DOI : https://doi.org/10.4000/add.1656 


\title{
L'avènement des peines alternatives en droit pénal camerounais : contours et concours de l'une des innovations de la réforme législative du 12 juillet 2016
}

\author{
Dieudonné SowENG
}

Parmi les peines principales qui répriment la commission des infractions en droit pénal camerounais, l'emprisonnement et l'amende figurent en très bonne place ${ }^{1}$. Ces sanctions ont certainement été retenues, au moment de leur consécration dans les codes pénaux successifs $^{2}$, compte tenu de la priorité accordée à l'efficacité de la répression qui a fondé pendant longtemps la politique pénale camerounaise ${ }^{3}$. Le

1. L. $\mathrm{n}^{\circ} 2016 / 007,12$ juillet 2016 , art. 18 portant code pénal: «Les peines principales sont: a) pour les personnes physiques : la peine de mort, l'emprisonnement, l'amende; b) pour les personnes morales : la dissolution, la fermeture temporaire ou définitive de l'établissement, l'amende."

2. Il s'agit des codes institués respectivement par la $\mathrm{L} . \mathrm{n}^{0} 65 / \mathrm{LF} / 24,12$ novembre 1965 (Journal officiel de la République fédérale du Cameroun - J.O.R.F.C. - 1965, $\mathrm{n}^{\mathrm{o}}$ sup., p. 85 ; R.C.D.C., t. v, p. 276-289) et la loi $\mathrm{n}^{\mathrm{o}} 67 / \mathrm{LF} / 1$ du 12 juin 1967 (J.O.R.F.C., $\mathrm{n}^{\circ}$ sup., p. 33 ; R.C.D.C., 1967, t. VII, p. 201-224, en ses art. 18 et 24). Ils étaient encore marqués par l'esprit de la répression de la grande criminalité, constituée de la rébellion d'après indépendance, qui a justifié l'intervention d'une série d'ordonnances de 1962 caractérisées par le peu de souci réservé aux droits fondamentaux du délinquant.

3. Jacqueline Costa-Lascoux, "Quelques aspects nouveaux du droit des pays africains d'expression française. Une politique pénale contre le sous-développement ", Penant, 1967 , p. 176 et suiv.; "Le droit pénal, l'unité nationale et le développement», Archives de politique criminelle, $\mathrm{n}^{\mathrm{o}} 1,1975$, p. 93-119; "Les politiques criminelles des États africains d'expression française ", dans Gérard Conac (dir.), Dynamique et finalités des droits africains, Paris, Economica, «La vie du droit en Afrique », 1980, p. 169-204; Dieudonné Célestin Mvogo, La politique criminelle au Cameroun: de la confrontation des modèles traditionnels et modernes à la recherche de solutions adaptées à un processus de développement, thèse de doctorat, université de Paris I, 1982; Adolphe Minkoa She, Essai sur l'évolution de la politique criminelle au Cameroun depuis l'indépendance, thèse de doctorat, Strasbourg III, 1987; Philippe Wakam, La protection des biens dans la politique criminelle du Cameroun, thèse de doctorat, université de Paris I, 1991 ; Elizabeth A. Nkongho, Evolution of Criminal 
système répressif d'alors visait davantage "à punir et non à corriger ${ }^{4}$ ". Le souci majeur était de sévir de telle manière que le délinquant ne soit pas en mesure, pendant l'exécution de la peine d'emprisonnement ou d'amende, de commettre de nouvelles infractions; d'autant plus que la sanction pécuniaire pouvait l'amener à craindre pour son patrimoine s'il persévérait dans l'infraction. La privation de liberté a en effet plusieurs objectifs sociaux, puisqu'elle permet de garder sous contrôle les personnes coupables d'infraction, ou soupçonnées d'en avoir commis une jusqu'à ce qu'un tribunal se prononce sur leur culpabilité. Elle sanctionne le délinquant condamné en le privant de liberté quand il est déclaré coupable et l'empêche ainsi d'en commettre davantage, tout en facilitant sa réadaptation pendant la période d'incarcération ${ }^{5}$. Dans le cadre de la justice pénale, le régime de la sanction concerne une partie des règles relatives au procès équitable dans laquelle le droit de l'accusé à une peine juste et humaine vise prioritairement sa réinsertion sociale ${ }^{6}$.

Cette option aurait certainement été l'idéal si l'administration pénitentiaire était dotée de moyens pouvant permettre que les peines soient suffisamment dissuasives, de manière à faire reculer la criminalité. Malheureusement, l'application des peines d'emprisonnement ou d'amende s'est davantage révélée être des prétextes servant à fouler aux pieds les droits des personnes condamnées. Les lieux et autres modalités d'exécution de leurs peines n'étaient pas propices à une bonne protection des droits de l'Homme. On se serait cru encore dans l'ancien droit où, pendant longtemps, les sanctions pénales étaient constituées de châtiments corporels, le condamné n'étant pas considéré comme un être humain à part entière ${ }^{7}$. Pourtant, dans la pratique, le recours systématique à

Laws in Cameroon: An Evaluation of the Dynamics of the Criminal Law Process, Ph.D. thesis, The Union Institute, 1996. Cités par Guy Blaise Dzeukou, Code de procédure pénale annoté et commenté, Bafoussam, Éditions juridiques camerounaises, 2007, t. 1, p. 27.

4. Bernard Bouloc, Pénologie, Paris, Dalloz, 2005, p. 3.

5. Ces fonctions de la sanction pénale restent actuelles même en droit français: art. 130-1 du Code pénal, «afin d'assurer la protection de la société, de prévenir la commission de nouvelles infractions et de restaurer l'équilibre social, dans le respect des intérêts de la victime, la peine a pour fonctions: 1) de sanctionner l'auteur de l'infraction; 2) de favoriser son amendement, son insertion ou sa réinsertion " (disposition du Code pénal telle que modifiée par l'art. $1^{\mathrm{er}}$ de la L. $\mathrm{n}^{\mathrm{o}} 2014-896 \mathrm{du}$ 15 août 2014 relative à l'individualisation des peines et renforçant l'efficacité des sanctions pénales).

6. Maria Stefania Cataleta, «Le principe de légalité de la peine en droit pénal international, points de force et de faiblesse », La Revue des droits de l'homme, no 9, 2016, http://revdh.revues.org/1868.

7. Ibid. 
l'emprisonnement augmente partout, sans que l'on puisse affirmer qu'il en résulte une amélioration de la sécurité publique ${ }^{8}$. Cette croissance refrénée du nombre de détenus aboutit en général à un grave surpeuplement carcéral, qui conduit à d'importantes violations des normes des Nations unies, en vertu desquelles tous les détenus doivent être traités avec le respect dû à leur dignité et à leur valeur intrinsèque en tant qu'êtres humains. La perte de liberté qui découle de l'emprisonnement entraîne aussi des atteintes à bien d'autres droits de l'homme, telles la privation de divers éléments de confort puisqu'ils vivent dans des cellules surpeuplées, la malnutrition, la vulnérabilité aux maladies et aux mauvais soins et même les difficultés à contacter leurs familles, etc. ${ }^{9}$ En outre, l'emprisonnement à outrance est contre-productif, puisqu'il n'est pas propice à la réadaptation et la réinsertion des personnes condamnées. De plus, il est inapte à préserver la présomption d'innocence dont doit normalement bénéficier la personne poursuivie et dont la culpabilité n'est pas encore établie.

Ici comme ailleurs, la faillibilité du système pénitentiaire a été démontrée et le besoin urgent de repenser la peine et ses effets sur la société contemporaine s'est imposé ${ }^{10}$. En effet, pour le détenu en attente de condamnation, parce qu'elle ne constitue pas encore une peine au sens plein, la privation de liberté doit être particulièrement justifiée, car jusqu'à preuve du contraire, le détenu est présumé innocent des charges qu'on veut lui imputer. Sa détention doit donc se justifier soit par le risque de fuite visant à éviter les poursuites, soit par le risque de dépérissement des preuves ou celui de l'intimidation des témoins potentiels. Cependant, elle peut en même temps avoir de graves inconvénients

8. En 2005, on estimait à 9 millions le nombre de détenus dans le monde; un chiffre sans cesse croissant. Voir Roy Walmsley, World Prison Population List, Londres, Institute for Criminal Policy Research.

9. Maurice Kamto, «L'énoncé des droits dans les constitutions des États africains francophones", R.J.A., 1991, $\mathrm{n}^{\circ}$ 2-3, p. 7-24; René Njeufack Temgwa, Le juge pénal et les droits de l'homme, mémoire de maîtrise, université de Dschang, 1998 ; Paul-Gérard Pougoué, "La législation camerounaise et la protection des droits de l'homme», Cahier africain des droits de l'homme, $\mathrm{n}^{\circ}$ 4, «Penser et réaliser les droits de l'homme en Afrique centrale », juillet 2000 ; Rose Kouayi Kemajou, Procès pénal et droits de l'homme dans le droit positif camerounais, thèse de doctorat, université Cheikh Anta Diop, Dakar, 2001; Michel Mahouvé, La protection des droits fondamentaux de la personne en droit extraditionnel camerounais, thèse de doctorat, université de Paris II, 2004.

10. Cette faillibilité a été amplement démontrée dans de nombreux pays par la doctrine. Voir Adalberto Carim Antonio, Les peines alternatives dans le monde, thèse de doctorat, université de Limoges, 2011, p. 433. 
pour l'ensemble du système de la justice pénale. Ainsi, la détention peut rendre plus difficile la préparation de la défense et entamer de manière plus générale l'efficacité de l'administration de la justice. De même, la probabilité de mise hors de cause de la personne poursuivie au bout de l'instruction est un facteur d'aggravation de la violation des droits du détenu provisoire; surtout lorsque l'on sait que l'article $236 \mathrm{du}$ Code de procédure pénale, qui institue une indemnisation en raison d'une détention provisoire ou d'une garde à vue abusive, n'a encore jusqu'ici reçu aucun commencement de mise en œuvre au Cameroun ${ }^{11}$. Pour le détenu condamné, on a déjà relevé le risque que les divers objectifs de l'emprisonnement ne soient pas atteints. Alors même qu'il est nécessaire que les objectifs de prévention de la commission de nouvelles infractions, de restauration de l'équilibre social ébranlé par la commission de l'infraction, de réhabilitation du délinquant et même de préservation des intérêts de la victime, soient tous atteints ${ }^{12}$.

Cette question des méfaits du recours excessif à l'emprisonnement ou l'amende est d'une telle acuité qu'il s'est avéré impérieux de rechercher des voies alternatives pouvant permettre, en même temps, d'atteindre les mêmes objectifs que ceux visés originellement par ces peines, de préserver la dignité du condamné en lui évitant les traitements inhumains et dégradants et de consacrer le droit d'aller et de venir de l'individu comme un droit fondamental de l'Homme ${ }^{13}$. La liberté individuelle est l'un des droits les plus fondamentaux de la personne humaine,

11. CPP, art. 236, 1: «Toute personne ayant fait l'objet d'une garde à vue ou d'une détention provisoire abusive peut, lorsque la procédure aboutit à une décision de non-lieu ou d'acquittement devenue irrévocable, obtenir une indemnité si elle établit qu'elle a subi du fait de sa détention un préjudice actuel d'une gravité particulière ( Samuel Tepi, «L'indemnisation des détentions provisoires abusives dans le nouveau Code de procédure pénale», Annales de la faculté des Sciences juridiques et politiques de l'université de Dschang, t. 11, 2007, p. 179). Voir aussi Dieudonné Soweng, «Regard comparatif sur les systèmes camerounais et français de réparation des privations abusives de liberté pendant le procès pénal», Annales de la faculté des sciences juridiques et politiques de l'université de Dschang, t. 14, 2010, p. 285-301.

12. À titre comparatif, c'est certainement ce qu'a prévu l'art. 130-1 du Code pénal français tel que modifié par l'art. $1^{\text {er }}$ de la L. $\mathrm{n}^{\mathrm{o}} 2014 / 896$ du 15 août 2014 relative à l'individualisation des peines et renforçant l'efficacité des sanctions pénales. https://www.legifrance.gouv.fr.

13. François Anoukaha, «La liberté d'aller et venir au Cameroun depuis le nouveau code de procédure pénale ", Annales de la faculté des sciences juridiques et politiques de l'université de Dschang, t. 11, 2007, p. 5-20. 
reconnue par les instruments internationaux des droits de l'Homme ${ }^{14}$ et la Constitution du Cameroun ${ }^{15}$. Pour priver quelqu'un de ce droit, même temporairement, les pouvoirs publics doivent pouvoir justifier le recours aux mesures privatives comme étant les seules susceptibles de permettre d'atteindre des objectifs qui ne peuvent être atteints par des moyens moins pernicieux. L'on estime en effet que la plupart des objectifs que poursuivait l'institution de la peine d'emprisonnement peuvent être atteints plus efficacement par d'autres moyens.

C'est dans cette mouvance qu'est née la nécessité de l'élaboration des sanctions dites alternatives à l'emprisonnement. Il s'agit de sanctions pénales qui peuvent être prononcées par le juge pénal en lieu et place de certaines sanctions dont la nocivité, sur des valeurs qui doivent rester protégées en dépit de la commission d'une infraction, a été démontrée. La faculté est ainsi offerte au juge pénal de substituer à l'emprisonnement ou à l'amende une peine d'une autre nature. C'est depuis le milieu des années 1950 que l'ONU a élaboré les premières règles et des normes ${ }^{16}$ pour encourager le développement de systèmes de justice pénale respectant les normes fondamentales relatives aux droits de l'homme.

Sur le plan international, les peines alternatives sont aujourd'hui recommandées par les «Règles minima des Nations unies (Règles de

14. Il s'agit de différents instruments auxquels le Cameroun a adhéré ou qu'il a ratifiés et qui consacrent à leur manière la liberté de mouvement de l'individu. Ce sont : la Déclaration universelle des droits de l'homme du 10 décembre 1948 dont les art. 3 et 9 prévoient respectivement que « tout individu a droit à la vie, à la liberté et à la sûreté de sa personne» et que «nul ne peut être arbitrairement arrêté, détenu ou exilé »; le Pacte international des Nations unies relatif aux droits civils et politiques du 16 décembre 1966 dont l'art. 9.1 dispose : «tout individu a droit à la liberté et à la sécurité de sa personne. Nul ne peut être privé de sa liberté, si ce n'est pour des motifs et conformément à la procédure prévue par la loi »; la Charte africaine des droits de l'homme et des peuples du 28 juin 1981, dont l'art. 6 est : «Tout individu a droit à la liberté et à la sécurité de sa personne. Nul ne peut être privé de sa liberté, sauf pour des motifs et dans les conditions préalablement déterminées par la loi ; en particulier, nul ne peut être arrêté ou détenu arbitrairement. »

15. Pour sa part, le préambule de la Constitution du 2 juin 1972 modifiée le 18 janvier 1996 admet que «la liberté et la sécurité sont garanties à chaque individu dans le respect des droits d'autrui et de l'intérêt supérieur de l'État» et que «nul ne peut être poursuivi, arrêté et détenu que dans les cas et selon les formes déterminées par la loi».

16. Résolution 663 C (XXIV) du Conseil économique et social du 31 juillet 1957 , document des Nations unies E/3048 (1957) et 2076 (LXII) (1957), citée par Dirk van Zyl Smit, Manuel des principes fondamentaux et pratiques prometteuses sur les alternatives à l'emprisonnement, Vienne, Office des Nations unies contre la drogue et le crime (ONUDC) ; New York, ONU, 2008, p. 10. 
Tokyo ${ }^{17}$ ». Ces règles présentent une série de recommandations qui insistent sur le fait que l'emprisonnement devrait être considéré comme un dernier recours. Elles encouragent, par conséquent, la promotion de mesures non privatives de liberté tout en veillant sur la préservation d'un juste équilibre entre les droits des délinquants, les droits de la victime et l'intérêt général de la société.

Sur le plan national au Cameroun, les mesures alternatives à la privation de liberté pendant le procès pénal existaient déjà implicitement à la phase de l'instruction préparatoire, sous la forme de mesures de surveillance judiciaire prévues par l'article 246 du Code de procédure pénale ${ }^{18}$. Cependant, les peines alternatives à proprement parler n'y ont fait une apparition explicite qu'avec l'avènement de la loi $n^{0}$ 2016/007 du 12 juillet 2016 portant Code pénal, dont l'article 18-1 dispose: «les peines alternatives sont: le travail d'intérêt général; la sanctionréparation ${ }^{19}$ ». Contrairement à ce qui est recommandé par les «Règles de Tokyo ", le législateur camerounais ne s'est pas contenté d'instituer des alternatives seulement à l'emprisonnement. Il a estimé que de telles mesures de substitution devaient exister également pour la peine

17. Ces règles minimales ont été examinées pour la première fois au $7^{\mathrm{e}}$ Congrès pour la prévention du crime et de la justice pénale et adoptées ensuite par l'AG de l'ONU, res. 45/110, 14 déc. 1990.

18. Art. 246 du Code de procédure pénal camerounais: "le juge d'instruction peut, par ordonnance, soumettre l'inculpé à des mesures de surveillance judiciaire ou substituer de telles mesures au mandat de détention provisoire, en l'astreignant, soit à une ou plusieurs des obligations [...], soit à une ou plusieurs de celles énumérées ci-après: a) Ne pas sortir des limites territoriales déterminées par le juge d'instruction; b) Ne pas se rendre en certains lieux déterminés par le juge d'instruction; c) Répondre aux convocations de toute autorité chargée de la mission de surveillance et d'assistance ou de toute autre personne désignée par le juge d'instruction; d) S'abstenir de conduire tous véhicules ou certains véhicules et, le cas échéant, remettre au greffe son permis de conduire contre récépissé; e ) S'abstenir de recevoir certaines personnes désignées par le juge d'instruction, ainsi que communiquer avec elles de quelque façon que ce soit ; f) Se soumettre à des mesures d'examen, de traitement ou de soins, même sous le régime de l'hospitalisation, notamment aux fins de désintoxication et de traitement des maladies contagieuses;

g) Fournir, en vue de garantir sa représentation en justice : soit un cautionnement dont le montant et les modalités de versement sont fixés par le juge d'instruction, compte tenu notamment des ressources de l'inculpé; soit un ou plusieurs garants $[\ldots]$; h) Ne pas exercer certaines activités professionnelles lorsque l'infraction a été commise à l'occasion ou dans l'exercice de celles-ci et si le juge d'instruction estime que leur poursuite est de nature à faciliter la commission d'une nouvelle infraction. »

19. Leur définition ainsi que leurs modalités d'application sont précisées aux art. 26, 26-1, 26-2 et 26-3 de ce Code pénal. 
d'amende, sanction qui s'exécute sur le patrimoine du délinquant ${ }^{20}$, un autre élément cher de la vie de ce dernier dont l'atteinte pourrait lui être gravement préjudiciable.

Au vu des objectifs qui sont poursuivis à travers l'instauration des peines alternatives par le législateur camerounais ${ }^{21}$, on devrait à terme connaître au minimum une stagnation et au plus une réelle diminution du nombre d'incarcérations, ainsi que des sanctions pouvant avoir un impact immédiat sur le patrimoine du condamné, sans qu'il y ait pour autant une entrave à l'efficacité de la répression des infractions. Il peut alors être pertinent de se demander si ces peines alternatives, telles qu'elles sont introduites en droit pénal camerounais, permettent d'atteindre les objectifs. Autrement dit, y a-t-il lieu d'espérer des améliorations sensibles dans la préservation des droits fondamentaux des condamnés à travers l'introduction, dans l'univers pénal camerounais, des peines alternatives?

La recherche de solutions adéquates à cette interrogation consistera à bien apprécier l'opportunité de l'introduction des peines alternatives dans l'arsenal répressif du Cameroun (1) et à mesurer l'impact de leur mise en œuvre sur une amélioration de son système de répression (2).

\section{L'opportunité de l'introduction des peines alternatives en droit pénal camerounais}

La question de l'amélioration du sort du délinquant au Cameroun peut-elle être résolue par la création de nouveaux types de peines qui lui sont infligées ${ }^{22}$ ? Plus explicitement, l'introduction des peines alternatives est-elle susceptible de concourir à une amélioration du système répressif camerounais? Ainsi se pose le problème de l'opportunité des peines alternatives qui doivent être une véritable réponse à la nécessaire fixation de sanctions pénales plus utiles (1.1), de même que leur introduction doit être suffisamment motivée (1.2).

20. Art. 26-1, 2, 3 du Code pénal : ils déterminent les modalités d’application des peines alternatives et prévoient qu'elles sont applicables à certaines infractions passibles d'un emprisonnement ou d'une peine d'amende.

21. Il ressort en effet de l'exposé des motifs du projet de loi $n^{\circ} 989 / \mathrm{PJL} / \mathrm{AN}$ portant Code pénal que «ces peines alternatives, dont l'application est encouragée par une directive générale des Nations Unies, visent à désengorger les prisons et permettent d'éviter les courtes peines d'emprisonnement ».

22. Marie-Ange Cochard, «La multiplication des peines : diversité ou dilution?», Revue juridique Droit pénal, $\mathrm{n}^{\circ}$ 9, septembre 2015, Lexis 360, dossier 9. 


\subsection{Une réponse à la nécessité de la détermination de sanctions pénales plus utiles}

L'expression "peines alternatives» n'est explicitement définie par aucun des textes qui les instituent. Le vocabulaire juridique définit littéralement la peine alternative comme une "peine qui peut être prononcée au lieu d'une autre et à titre de peine principale ${ }^{23} »$. La règle 2.3 des "Règles minima des Nations unies pour l'élaboration de mesures non privatives de liberté» prévoit, entre autres, que "pour que soit évité un recours inutile à l'incarcération, le système de justice pénale devrait prévoir un vaste arsenal de mesures non privatives de liberté, depuis les mesures pouvant être prises avant le procès jusqu'aux dispositions relatives à l'application des peines. Le nombre et les espèces de mesures non privatives de liberté disponibles doivent être déterminés de telle manière qu'une fixation cohérente de la peine demeure possible». Cet instrument international semble ne proposer aucune définition de la peine alternative et ne préconise donc aucune énumération limitative ou exhaustive des mesures alternatives qu'il recommande aux États. Chaque État est simplement appelé à prévoir dans son système pénal « un vaste arsenal de mesures non privatives de liberté » en fonction des objectifs liés à la recherche d'un relatif équilibre entre les droits du délinquant, les prérogatives reconnues à la victime et l'intérêt général de la société. Bien que l'instauration des mesures alternatives soit fortement recommandée, les États conservent la faculté d'adopter celles qui leur conviennent puisque la règle 8.2 utilise le verbe "pouvoir", sans doute, pour indiquer le caractère non contraignant et non limitatif des mesures qui sont proposées aux autorités compétentes de chaque État ${ }^{24}$.

Les systèmes pénaux ne sont pas obligés de qualifier de telles mesures de "peines alternatives»; elles peuvent être dénommées autrement et

23. Association Henri Capitant, Gérard Cornu (dir.), Vocabulaire juridique, Paris, PUF, 2013, p. 749 ( $10^{\mathrm{e}}$ éd.).

24. Il ressort de la règle 8.2 des règles de Tokyo que «Les autorités compétentes peuvent prendre les mesures suivantes: a) Sanctions orales, comme l'admonestation, la réprimande et l'avertissement; b) Maintien en liberté avant décision du tribunal; c) Peines privatives de droits; d) Peines économiques et pécuniaires, comme l'amende et le jour-amende; e) Confiscation ou expropriation; f) Restitution à la victime ou indemnisation de celle-ci; g) Condamnation avec sursis ou suspension de peine; h) Probation et surveillance judiciaire; i) Peines de travail d'intérêt général ; j) Assignation dans un établissement ouvert; k) Assignation à résidence ; 1) Toute autre forme de traitement en milieu libre; $m$ ) Une combinaison de ces mesures.» 
conserver la même fonction. En droit pénal français, elles ont pendant longtemps été qualifiées dans la législation de "peines de substitu$\operatorname{tion}^{25}$ ", entendues comme des peines que le juge prononce au lieu de l'emprisonnement normalement applicable, partant de l'idée que cette dernière peine serait inadaptée et que la peine de substitution aurait des chances d'être plus efficace. D'ailleurs, le texte des Nations unies semble n'envisager de peines alternatives qu'à l'emprisonnement alors même que dans certains systèmes répressifs, comme celui du Cameroun, il est possible, avec la nouvelle législation de 2016, de prononcer des sanctions alternatives à la peine d'amende. Ce qui peut amener à s'interroger sur l'objectif poursuivi par le législateur camerounais à travers l'instauration des peines alternatives. En effet, on peut aisément comprendre que les alternatives à l'emprisonnement visent à résoudre le problème du surpeuplement des établissements pénitenciers, celui de son coût financier et celui des violations des droits des personnes condamnées à l'incarcération. Mais on peine à trouver une explication fiable et irréfutable à l'instauration des alternatives à l'amende qui, dans les «Règles de Tokyo", constituerait elle-même une mesure alternative à l'emprisonnement ${ }^{26}$.

Le nouveau Code pénal camerounais prévoit quatre catégories de peines : les peines principales ${ }^{27}$, les peines alternatives, les peines accessoires ${ }^{28}$ et

25. Loi française du 11 juillet 1975 créant des substituts à la peine de l'emprisonnement ainsi que la possibilité de reconnaître la culpabilité du délinquant en l'exemptant cependant de toute peine, citée dans Gaston Stéfani, George Levasseur et Bernard Bouloc, Droit pénal général, Paris, Dalloz, 2005, p. 94. Voir André Decocq, «Les modifications apportées par la loi du 11 juillet 1975 à la théorie générale de droit pénal », Revue de science criminelle, 1976, p. 5 ; Paul-Julien Doll, «Analyse des dispositions essentielles des lois 75-624 du 11 juillet 1975 et 75-701 du 6 août 1975 », Gazette du Palais, t. 1, 1976, p. 34, 39 et 104; Jean Robert, «Les lois du 11 juillet et du 6 août 1975 en matière pénale ", J.C.P., 1975.

26. La règle $8.2 \mathrm{~d}$. des règles de Tokyo prévoit, entre autres mesures alternatives à l'emprisonnement, que le juge peut prononcer les "peines économiques et pécuniaires, comme l'amende et le jour-amende».

27. Parmi les peines principales, on retrouve pour la personne physique, la peine de mort, l'emprisonnement et l'amende; pour les personnes morales, la dissolution, la fermeture temporaire ou définitive de l'établissement et l'amende (C. pén., art. 18).

28. Les peines accessoires sont constituées pour les personnes physiques des déchéances, de la publication de la décision, de la fermeture de l'établissement et de la confiscation; pour les personnes morales, il s'agit de l'interdiction, pour une durée déterminée, de s'investir directement ou indirectement dans l'une ou plusieurs des activités prévues par son objet social, du placement sous surveillance judiciaire pendant une durée déterminée, de la fermeture, pour une durée déterminée, des établissements ou succursales ayant servi à la commission des faits incriminés et de 
les mesures de sûreté ${ }^{29}$. Aux termes de l'article 18-1 de ce Code pénal, les peines alternatives qui ont été retenues par ce législateur pénal sont au nombre de deux : le travail d'intérêt général et la sanction-réparation.

Concernant le travail d'intérêt général, l'article 26 de la loi du 12 juillet 2016 portant Code pénal en fixe les modalités, sans toutefois lui donner une définition explicite. Mais selon la doctrine, il consiste en « une prestation d'activité non rémunérée accomplie au profit d'une collectivité publique, d'un établissement public ou d'une association ${ }^{30}$ ». Pour la loi camerounaise, le bénéficiaire peut être, soit une personne morale de droit public, soit une personne morale de droit privé chargée d'une mission de service public, ou encore un organisme habilité à mettre en œuvre des travaux d'intérêt général. Le travail d'intérêt général peut consister, par exemple, en des travaux d'amélioration de l'environnement, d'entretien ou de réparation. En France, le travail d'intérêt général peut également être assorti d'une peine d'emprisonnement avec sursis applicable en cas de non-respect de certaines obligations l'assortissant. Ce n'est pas le cas en droit camerounais où l'article 26 (5), en prescrivant au juge de prévoir la durée de l'emprisonnement encouru en cas d'inexécution de la peine de travail d'intérêt général, interdit formellement de l'assortir de sursis. Plutôt que de confisquer la liberté du délinquant par le prononcé d'un emprisonnement ou de préjudicier à son patrimoine par une condamnation à une peine d'amende, le juge peut donc se borner à réduire sa liberté à travers l'imposition d'une activité déterminée sans contrepartie.

Le bénéficiaire de ce travail d'intérêt général étant l'ensemble de la collectivité, ou tout au moins une partie de celle-ci, le travail d'intérêt général fait partie de ce que l'on peut qualifier de peine citoyenne, car comme son nom l'indique, il doit servir l'intérêt général. Avant de prononcer cette sanction, on doit donc s'assurer des retombées collectives et surtout qu'un tel travail peut être exécuté sous une surveillance appropriée. Ce travail exige en effet une surveillance étroite, afin de

la publication de la décision ou sa diffusion par voie de médias et de toutes autres peines accessoires prévues par les textes spéciaux (C. pén., art. 19).

29. Les mesures de sûretés quant à elles sont constituées pour les personnes physiques de l'interdiction de l'exercice de la profession, de la relégation, des mesures de surveillance et d'assistance postpénales, de l'internement dans une maison de santé et de la confiscation; pour les personnes morales, de l'interdiction de s'investir dans une activité précise pour une durée déterminée, de la confiscation et du placement sous surveillance judiciaire pour une durée déterminée (C. pén., art. 20).

30. Jean Pradel, Droit pénal général, Paris, Cujas, 2014, p. 551, $\mathrm{n}^{\circ} 659$. Voir Vincent Delbos, «Travail d'intérêt général », Répertoire de droit pénal et de procédure pénale, juin 2013, p. 2. 
vérifier que le condamné exécute effectivement la tâche imposée, et qu'il n'est pas contraint à travailler pour une fin autre ou dans des conditions de nature à porter atteinte à sa dignité.

Le travail d'intérêt général est donc une modalité particulière de sanction pénale, substitut à l'emprisonnement ou à l'amende, au service de la communauté, inspiré par le succès du community service order en Grande-Bretagne. Introduis en droit français par une loi du 10 juin $1983^{31}$, il est instauré en droit camerounais récemment par le Code pénal du 12 juillet 2016, en conformité avec la recommandation du point 8.2 i des « Règles minima des Nations unies » évoquées ci-dessus ${ }^{32}$. À la question de savoir pourquoi introduire dans le droit positif le travail d'intérêt général, Jean Pradel répond que «la raison est le souci de réduire la nocivité ${ }^{33}$ des courtes peines d'emprisonnement ${ }^{34}$ ». La pertinence de cette raison est telle que l'instauration du travail d'intérêt

31. L. $\mathrm{n}^{0} 83-466$, D. $\mathrm{n}^{0} 1983-282$, votée à l'unanimité par les parlementaires; L. $n^{\circ} 87-519, D . n^{\circ} 1987-286,10$ juill. 1987, fait du travail d'intérêt général une peine complémentaire en matière d'infractions à la circulation routière; L. $\mathrm{n}^{0} 89-461$, D. $\mathrm{n}^{\circ}$ 1989-224, 6 juill. 1989, permet la saisine de la juridiction de condamnation pour prononcer le sursis à l'exécution de la peine d'emprisonnement assorti de l'obligation d'effectuer un TIG (travail d'intérêt général), lorsque le jugement est prononcé hors de la présence du prévenu (procédure dite de la « conversion en sursis assortie de l'obligation d'effectuer un travail d'intérêt général » (C. pén., art. 132-57). Les lois des 22 juillet et 16 décembre 1992 instaurant le Code pénal actuel modifient les textes alors applicables. La loi du 8 février 1995, modifiant l'art. 132-57 du Code pénal (L. no $95-125$, D. 1995-178), vient ouvrir la possibilité d'une «conversion sursis-travail d'intérêt général» aux peines d'emprisonnement prononcées en présence du prévenu. La loi du 9 septembre 2002 a instauré la possibilité d'un cumul entre une peine d'amende et un travail d'intérêt général dans le cadre de certaines dégradations aux biens. Voir Gaston Stefani, George Levasseur et Bernard Bouloc, op. cit., p. 496; Pierre Couvrat, «Les trois visages du travail d'intérêt général », RSC, 1989, p. 158 et suiv.; Jean Pradel, "Les nouvelles alternatives à l'emprisonnement créées par la loi no 83-466 du 10 juin 1983", D., 1984, p. 37 et Jean-Marc Puech, "Commentaires de la loi n ${ }^{\circ} 83-466$ du 10 juin 1983 ", A.L.D., 1983, p. 105 et suiv.; Jacques Vérin, «À la recherche de vrais substituts à l'emprisonnement », RSC, 1982, p. 399 et suiv.

32. Le point $8.2 \mathrm{i}$ de ces "Règles minima des Nations unies" propose comme autres peines alternatives que le juge peut prononcer les "peines de travail d'intérêt général».

33. Cette nocivité était déjà dénoncée au détbut du $\mathrm{xx}^{\mathrm{e}}$ siècle par l'Allemand Franz von Liszt (Traité de droit pénal allemand, René Lobstein trad., Paris, V. Giard et E. Brière, 1911, t. 1, p. 108).

34. Jean Pradel, op. cit., p. 551. 
général a aujourd'hui tendance à se généraliser dans de nombreuses législations ${ }^{35}$.

Malgré la pertinence de son entrée dans le paysage juridique camerounais, on peut déplorer le fait que les juridictions sont encore dans l'impossibilité de prononcer cette sanction, faute de la détermination explicite de son contenu. À travers les dispositions de l'article 26, alinéa 6 du nouveau Code pénal, le législateur réserve, en effet, l'établissement de la liste des travaux d'intérêt général à un texte particulier ${ }^{36}$. Nul doute qu'il s'agira d'un texte réglementaire. Ce qui risque d'être en contradiction avec les recommandations des "Règles de Tokyo », qui reconnaissent le danger de telles peines et demandent, en des termes péremptoires, que «l'adoption, la définition et l'application de mesures non privatives de liberté doivent être prescrites par la loi ${ }^{37} »$. Sauf à opter pour une assertion large de la notion de loi, il aurait été plus indiqué pour le législateur d'énumérer dans la loi portant Code pénal les activités pouvant faire l'objet de travail d'intérêt général. Les « Règles de Tokyo » ont certainement voulu, dans l'esprit même du principe sacré de la légalité des peines ${ }^{38}$, reconnaître une compétence exclusive à la loi dans son sens strict et donc limiter le pouvoir d'autres entités publiques, afin d'éviter la création et l'imposition de ce que l'on appelle des peines sur mesure, c'est-à-dire des peines non privatives de liberté qui sont uniques et ne découlent pas d'un cadre pénal établi. Une définition juridique des

35. Par exemple, il est instauré en Grande-Bretagne depuis 1972 sous le nom de community service order, aux États-Unis et au Québec sous la dénomination de «travaux communautaires», au Portugal (C. pén., art. 6o, 1982) et dans certains pays nordiques. En France, cette sanction avait été recommandée par deux groupes d'études: le comité d'étude sur la violence, dit comité Peyrefitte (1977) et la commission des maires sur la sécurité, dite commission Bonnemaison (1982). Les pays africains ne sont pas en reste; ainsi le Zimbabwé, par une loi adoptée en 1992, modifiait le Code de procédure pénale pour permettre aux tribunaux, entre autres possibilités, d'ordonner un travail d'intérêt général.

36. Ce texte pourrait se référer éventuellement à la liste ci-après: amélioration de l'environnement, reboisement, débroussaillage des forêts, nettoyage des plages, réparation des dégâts causés par des actes de vandalisme, entretien des bâtiments publics (peinture, menuiserie, électricité, réparation des écoles), aménagement des espaces publics (parcs publics, stades), prestations au sein des services administratifs des personnes bénéficiaires, tâches de classement, d'archivages, de manutention ou d'entretien des équipements.

37. Règle 3.1 des Nations unies, déjà citée.

38. Michel Véron, "Principe de légalité criminelle - peine complémentaire et de substitution: la double erreur», commentaire sous Cass. crim. 13 avril 2010, $\mathrm{n}^{\mathrm{o}}$ 09-84.583, R-P+F: Juris Data $\mathrm{n}^{\circ}$ 2010-005942, Revues juridiques Droit pénal, $\mathrm{n}^{\mathrm{o}}$ 7-8, juillet 2010, comm. 81. Voir Maria Stefania Cataleta, art. cité. 
peines alternatives à l'emprisonnement permet aussi d'éviter des excès dans des peines par ailleurs acceptables.

La sanction-réparation quant à elle consiste, aux termes de l'article 26-1, alinéa 1 de la loi du 12 juillet 2016 portant Code pénal camerounais, «dans l'obligation, pour le condamné, de procéder à la réparation matérielle du préjudice subi par la victime ». Elle peut être prononcée par la juridiction de jugement à la place de l'emprisonnement ou de l'amende après la déclaration de culpabilité. La consécration de la sanctionréparation par le législateur camerounais vise à conformer sa législation, comme dans d'autres pays ${ }^{39}$, à la règle 8.2.f des "Règles de Tokyo", qui inclut parmi les mesures alternatives à l'emprisonnement que le juge peut prendre, la «restitution à la victime ou (l')indemnisation de celle-ci ». En raison de sa dénomination, la sanction-réparation semble provenir d'une alchimie a priori indigeste entre le droit pénal et le droit civil. Elle est à la fois une peine au sens pénal du terme et un procédé d'indemnisation civile de la victime, donc une mesure réparatrice et pas seulement répressive. Elle se présente donc selon deux modalités: indemnisation du préjudice issu de l'infraction ou réparation en nature consistant en la remise en l'état réalisée par le condamné lui-même.

Avec l'instauration de la sanction-réparation, la distinction des responsabilités pénale et civile perd encore un peu plus de sa netteté ${ }^{40}$. La confusion surtout des fonctions des responsabilités pénales et civiles peuvent même amener à s'interroger sur la dénomination de la peine; s'agit-il de la réparation-sanction ou de la sanction-réparation? Cette interrogation semble importer peu. Ce qui est constant, c'est que, ainsi que l'a affirmé Philippe Conte «le coupable d'une faute pénale n'est pas traité autrement que l'auteur d'une faute civile ${ }^{41} »$. Pour Jean-

39. En France, la sanction-réparation a été créée par une loi du 5 mars 2007; de même, la tradition est en faveur de l'indemnisation, des travaux menés au Nigéria et dans d'autres pays d'Afrique montrent qu'il existe une tradition ancienne consistant à indemniser les victimes au lieu de punir les délinquants, même pour les infractions les plus graves. Cette indemnisation est souvent versée en dehors de toute action judiciaire formelle, et le droit pénal n'est pas du tout invoqué. Cela tient au fait que le droit pénal n'est pas suffisamment souple pour reconnaître la nécessité d'une indemnisation. Voir Adedokum A. Adeyemi, «Personal reparation in Africa : Nigeria and Gambia» dans Alternatives to Imprisonment in Comparative Perspective, Ugljesa Zvekic (dir.), Chicago, Nelson, 1994, p. 53 et suiv.

40. Jean Pradel, op. cit., p. 553.

41. Philippe Conte, «La loi sur la prévention de la délinquance (loi $\mathrm{n}^{0}$ 2007-297 du 5 mars 2007) : présentation des dispositions de droit pénal ", Droit pénal, $\mathrm{n}^{\circ}{ }_{5}, 2007$, p. 12 et suiv. 
Christophe Saint-Pau, la compassion pour les victimes d'infraction pénale conduit à leur accorder une plus grande place dans le procès pénal auquel elles sont en principe accessoires, et au plan substantiel à conférer un effet réparateur à la condamnation pénale; ce phénomène est patent avec l'institution de la sanction-réparation ${ }^{42}$. Le fait pour le juge pénal de pouvoir ordonner la réparation du préjudice subi par la victime d'une infraction, en présence ou en l'absence d'une condamnation à une peine, n'est pas une révolution en soi. Par contre, c'est véritablement une nouveauté, en droit pénal camerounais de qualifier l'obligation de réparer de sanction à caractère pénal parce qu'imposée par la loi pénale.

D'un point de vue pratique, cette nouvelle sanction est donc la bienvenue pour les victimes qui peuvent être restaurées dans leurs droits, alors même qu'elles ne s'étaient pas constituées parties civiles ${ }^{43}$ et dont le règlement de la créance a de fortes chances d'être diligent, car elle est accolée à une sanction pénale. Le contexte camerounais est parfois caractérisé par l'ignorance de la victime quant aux précautions à prendre pour se constituer partie civile. On peut imaginer quelle sera la satisfaction d'une telle victime de savoir qu'elle pourra désormais être restaurée dans ce qu'elle a perdu du fait de la commission de l'infraction, à la suite d'une question orale du juge pour s'assurer que le délinquant marque son accord à la réparation, puisque cet accord doit toujours être requis ${ }^{44}$, conformément à la règle 3.4 des «Règles de Tokyo" selon laquelle «Les mesures non privatives de liberté qui entraînent une obligation pour le délinquant [...], requièrent le consentement du délinquant $»$.

L'admission de la restitution et de l'indemnisation permet de poursuivre un des objectifs de la justice pénale. Il est généralement admis que les dispositions relatives à la victime y occupent une place importante, car la Déclaration des principes fondamentaux de justice relatifs aux victimes de la criminalité et aux victimes d'abus de pouvoir prévoit

42. Jean-Christophe Saint-Pau, «La responsabilité pénale réparatrice et la responsabilité civile punitive», Responsabilité civile et assurances, $\mathrm{n}^{\circ}{ }_{5}$, mai 2013, Lexis 360 , dossier 23; David Dechenaud, «Les concours de responsabilité civile et de responsabilité pénale», Responsabilité civile et assurance, $\mathrm{n}^{\circ}{ }_{2}$, février 2012, Lexis 360 , dossier 5 .

43. Guy Blaise Dzeukou et Darly Aymar Djofang, «L'acquisition, par la victime, de la qualité de partie au procès pénal au regard du Code de procédure pénale et de la jurisprudence de la Cour suprême", Annales de la FSJP, t. 14, 2010, p. 271-291.

44. L'exposé de motif de la loi camerounaise du 12 juillet 2016 portant Code pénal admet, en effet, à son art. 26-1 qu' « en pratique, le condamné accepte de procéder à la réparation matérielle du préjudice de la victime». 
que «les auteurs d'actes criminels ou les tiers responsables de leur comportement doivent, en tant que de besoin, réparer équitablement le préjudice causé aux victimes, à leur famille ou aux personnes à leur charge ${ }^{45}$ ».

L'application de la sanction-réparation peut varier en fonction des hypothèses. Ainsi, dans l'hypothèse d'une infraction continue qu'il convient de faire cesser, la sanction pourrait consister à imposer une prestation précise au condamné avec astreinte ${ }^{46}$. Celle-ci visant à lui faire exécuter la prestation le plus diligemment possible. Dans son contenu réel, la sanction-réparation pourra consister en la remise en l'état d'un lieu ou d'une situation qui avait été perturbée par l'infraction. Il s'agit d'une sorte de restitutio in integrum ou restitution, ou même de la cessation immédiate d'une activité illicite. Elle peut également consister en la remise d'un document, d'une pièce ou une sorte d'actio exhibendum. La réparation due à la victime doit inclure la restitution des biens, une indemnité pour le préjudice ou les pertes subies, le remboursement des dépenses engagées en raison de la victimisation, la fourniture de services et le rétablissement des droits ${ }^{47}$.

Les peines alternatives, telles que déterminées par le nouveau Code pénal camerounais, se veulent donc plus utiles. Plus utiles aux individus, au condamné qui sera sanctionné, sans pour autant être autrement humilié, pour l'infraction dont il s'est rendu auteur et à la victime dont le préjudice découlant de l'infraction pourra être plus diligemment réparé. Elles seront également plus utiles à la société, qui pourra bénéficier de retombées consistantes issues par exemple du travail d'intérêt général et aussi à l'État dont l'image pourra être mieux soignée, si du fait

45. Art. 8 de la Déclaration des principes fondamentaux de justice relatifs aux victimes de la criminalité et aux victimes d'abus de pouvoir, cité par Dirk van Zyl Smit, op. cit., p. 34 .

46. Sur sa nature juridique, on s'est généralement demandé si l'astreinte, accompagnant la condamnation à une prestation en nature, est bien une sanction. La jurisprudence française décide que l'astreinte prononcée par le juge pénal «n'est ni une peine complémentaire, ni une peine accessoire, mais une condamnation pécuniaire prononcée à titre comminatoire à l'encontre du débiteur d'une obligation de faire ou de donner pour le contraindre à exécution » (Cass. crim. 10 février 1964, D. 1964, 376, note Jacques Mazard ; Cass. crim.7 décembre 1972, D. 1973, 697, note Bernard Bouloc, ou Cass. crim. 25 avril 1988, R.C. $\mathrm{n}^{\circ} 171$ ). Si dans un premier temps, l'astreinte est un moyen de contrainte, elle devient, après sa liquidation, une vraie peine, une peine pécuniaire dont le montant sera versé au Trésor public. Dans ce sens, voir Jean Pradel, op. cit., p. 555.

47. Art. 8 de la Déclaration des principes fondamentaux de justice relatifs aux victimes de la criminalité et aux victimes d'abus de pouvoir. 
d'une réduction de la population carcérale les conditions d'exécution des peines privatives de liberté venaient à s'améliorer.

Cela rend plus pertinente la motivation de l'introduction par le législateur des peines alternatives au Cameroun.

\subsection{Une introduction motivée des peines alternatives en droit pénal camerounais}

L'introduction des peines alternatives dans un système pénal doit être de nature à permettre la résolution d'un certain nombre de problèmes que posent les peines classiques. Ces solutions peuvent avoir des motivations juridiques, économiques ou sociales.

Sur un plan purement juridique, on a eu à dénoncer les menaces d'atteintes aux droits fondamentaux des condamnés aux peines privatives de liberté ou à certaines sanctions pécuniaires. De plus, les Nations Unies font le constat selon lequel « le recours général à l'emprisonnement augmente partout, sans que l'on puisse affirmer qu'il en résulte une amélioration de la sécurité publique ${ }^{48}$ ». Pourtant, l'objectif majeur devrait être celui d'allier la sévérité de la sanction pénale à l'atteinte d'une certaine efficacité de la répression. La réalité de l'accroissement du nombre de détenus conduit généralement à un grave surpeuplement carcéral, ce qui se traduit par des conditions d'incarcération qui violent les normes des Nations Unies et autres normes qui exigent que tous les détenus soient traités avec le respect dû à leur dignité et à leur valeur intrinsèque en tant qu'êtres humains. D'ailleurs, le préambule de la Constitution du Cameroun ${ }^{49}$ affirme clairement que « toute personne a droit à la vie et à l'intégrité physique et morale. Elle doit être traitée en toute circonstance avec humanité. En aucun cas, elle ne peut être soumise à la torture, à des peines ou traitements cruels, inhumains ou dégradants ». Cette exigence peut dès lors justifier la nécessité de recourir aux peines alternatives qui visent à réduire le nombre de personnes incarcérées, de telle manière que l'emprisonnement ne reste qu'une solution de dernier recours.

La liberté individuelle constitue l'un des droits les plus fondamentaux de la personne humaine, reconnue par les instruments internationaux des droits de l'homme que le Cameroun a ratifiés ainsi que par sa

48. Dirk van Zyl Smit, op. cit., p. 3 .

49. Cette constitution est issue de la L. $\mathrm{n}^{\circ}$ 96/o6 du 18 janvier 1996 portant révision de la Constitution du 2 juin 1972. 
Constitution ${ }^{50}$. La privation de cette liberté, même à titre temporaire par les pouvoirs publics, à travers le recours à l'emprisonnement, doit être justifiée comme étant une mesure nécessaire pour atteindre un objectif sociétal important, qui ne peut l'être par des moyens moins restrictifs. En effet, l'emprisonnement n'entraîne pas seulement la perte de liberté, il constitue aussi une atteinte à bien d'autres droits de la personne humaine. Au Cameroun, comme dans de nombreux autres pays, les prisonniers sont généralement privés de tout élément de confort, subissent le surpeuplement excessif des cellules, la malnutrition autant qu'ils sont mal vêtus et mal soignés, tout en subissant l'isolement dans leurs relations avec leur famille et leurs proches ${ }^{51}$. De tels traitements sont constitutifs de graves atteintes à la dignité humaine puisqu'ils se rapprochent de ce qu'il est convenu d'appeler «traitement inhumain et dégradant». Les peines alternatives devraient permettre de réduire la surpopulation carcérale ${ }^{52}$ et faciliter en conséquence la gestion des lieux de détention, afin que l'État puisse s'acquitter des obligations qui lui incombent vis-à-vis des personnes dont la privation de liberté aura été inévitable. L'objectif devant être une humanisation maximale du traitement du délinquant, de telle manière qu'il ne se sente pas marginalisé et qu'il ait la conviction de rester un membre de la société pour laquelle il devrait encore être utile.

La finalité sociale, quant à elle, relève de l'objectif de rééducation et de resocialisation du condamné visé par la règle 1.5 des «Règles de Tokyo", qui fait de la rationalisation des politiques de justice pénale, eu égard aux besoins de réinsertion sociale du délinquant,

50. Le préambule de cette Constitution affirme l'attachement du peuple camerounais "aux libertés fondamentales inscrites dans la Déclaration universelle des droits de l'homme, la Charte des Nations Unies, la Charte africaine des droits de l'homme et des peuples et toutes les conventions internationales y relatives et dûment ratifiées ».

51. François Betfian Yonou, Pauvreté, délinquance et sa répression au Cameroun, thèse de doctorat, université de Rennes I, 1977; Marthe Bornecque, La criminalité à Yaoundé et son contrôle, thèse de doctorat, université d'Aix Marseille III, 1979; Ajanoh Sone, The Treatment of Offenders in Cameroon, thèse de doctorat, université de Yaoundé, 1985 ; Jacques Sese, Les aspects criminologiques de la délinquance au Cameroun (particulièrement chez les Bantous-Semi-Bantous au sud et les Matakam au nord), thèse de doctorat, Strasbourg III, 1989.

52. L'efficacité des peines alternatives a été prouvée par exemple au Malawi qui, pour réduire sa population carcérale, a lancé en 2000 un plan concernant les peines de travail d'intérêt général. À la fin de septembre 2004, 5225 délinquants exécutaient de telles peines. Ils ont accompli 838 ooo heures de travail et achevé $87 \%$ des tâches qui leur étaient assignés. De même, pour les délinquants qui se sont acquittés de leur obligation de travail d'intérêt général, le taux de récidive est tombé à 0,25\%, soit un pour 400 délinquants. Voir Dirk van Zyl Smit, op. cit., p. 79. 
une des prescriptions majeures aux États, car «il a été montré que l'emprisonnement était contre-productif pour la réadaptation et la réinsertion des personnes accusées de délits mineurs, ainsi que pour certaines populations vulnérables ${ }^{53}$ ». D’ailleurs, le risque de récidive ou d'aggravation de la dangerosité du prisonnier est souvent élevé lorsque celui-ci purge sa peine dans des conditions carcérales peu propices à sa pénitence ou à son repentir. Adalberto Carim Antonio n'affirme-t-il pas qu'historiquement, il n'y a pas de rapports importants d'expériences réussies dans la réhabilitation des prisonniers subissant les rigueurs de la prison ${ }^{54}$ ?

En droit français, il est explicitement admis que la deuxième fonction de la sanction pénale infligée au délinquant est, selon l'article 130-1 du Code pénal, modifié par l'article 1 de la loi du 15 août 2014, "de favoriser son amendement, son insertion ou sa réinsertion». Par contre, au Cameroun, tout semble indiquer que la priorité n'est paradoxalement pas au détenu et à sa réinsertion sociale. Cela est dû, dans ce pays, à la promiscuité, conduisant à la proximité avec des facteurs criminogènes des milieux carcéraux ${ }^{55}$. Malgré les dispositions légales impératives qui prescrivent que les conditions d'exécution des peines privatives de liberté doivent «tenir compte de la nature de l'infraction, du quantum de la peine, du sexe, de l'âge, de l'état de santé mentale ou physique et de la conduite du condamné, de manière à concilier la nécessité de la réinsertion sociale et les impératifs de la discipline ${ }^{56}$ ", il est courant que les mineurs purgent leur peine dans les mêmes locaux que les adultes, les délinquants primaires au même lieu que les multirécidivistes ou de dangereux criminels parfois condamnés à perpétuité ou à mort. Il y a souvent une absence de locaux spécifiques aux détenus en attente de jugement et dont la culpabilité n'est pas encore établie. Dans la réalité, les mineurs et les femmes ne sont logés que dans des quartiers théoriquement spécifiques; le cloisonnement entre ces quartiers du même

53. Dirk van Zyl Smit, op. cit., p. 3.

54. Adalberto Carim Antonio, op. cit., p. 11.

55. Jacques Oberlin Mbock, La prison camerounaise (Étude critique de la réforme pénitentiaire de 1973 et de son application), thèse de doctorat, université Yaoundé, 1989; Vieux Eyike, "Les droits du prisonnier», Cahiers africains des Droits de l'homme, $\mathrm{n}^{\circ}$ 1, novembre 1998 ; Bernard-Raymond Guimdo Dongmo, "Les alternatives à l'emprisonnement dans des contextes de surpeuplement carcéral: le cas du Cameroun ", Juridis périodique, $\mathrm{n}^{\circ}$ 60, 2004, p. 77-85.

56. Art. 555, al. 2, Code de procédure pénale camerounais. Voir D. $\mathrm{n}^{\circ}$ 92-052, 27 mars 1992, dans Guy Blaise Dzeukou, op. cit., t. 1, p. 473 et 482. 
établissement pénitencier étant très lâche, sans pouvoir empêcher efficacement des contacts préjudiciables à la recherche d'une amélioration de la moralité des détenus. Pourtant, à l'individualisation des peines à prononcer devrait aussi correspondre une sorte d'individualisation des modalités d'exécution des peines; toutes choses qui devraient concourir à un réel renforcement de l'efficacité des sanctions pénales.

Les Nations unies, à travers l'Office contre la drogue et le crime, illustrent parfaitement ce risque en affirmant que :

Dans le monde entier, la majorité des prisonniers sont issus de milieux défavorisés tant économiquement que socialement. Pauvreté, chômage, logements insalubres, foyers brisés, troubles psychologiques et maladie mentale, toxicomanie et alcoolisme, violence conjugale: autant de réalités quotidiennes pour la plupart des délinquants. Beaucoup sont en prison pour des délits mineurs, ou non violents. En recourant à la prison comme réponse à tous les délits commis par ces personnes vulnérables, non seulement on ne résout pas la question de la sécurité dans nos communautés de manière durable, mais encore perpétue-t-on le cycle d'appauvrissement, de chômage, d'amenuisement des chances de trouver un emploi, de couples brisés, d'aggravation des troubles psychologiques et mentaux et des problèmes de toxicomanie. De plus, la surpopulation carcérale s'accompagne de nombreux risques pour la santé, dont la transmission de maladies infectieuses, plus particulièrement la tuberculose et le VIH. Dans bien des pays, la violence est le lot quotidien des prisonniers, plus encore dans les prisons surpeuplées ${ }^{57}$.

L'instauration des peines alternatives sera de nature à minimiser le risque d'augmentation du taux de criminalité et de récidive. L'auteur Adalberto Carim Antonio constate fort à propos qu' «une multitude d'études indique un faible taux de récidive chez les délinquants condamnés à des peines alternatives en comparaison de ceux qui ont été soumis au processus traditionnel d'emprisonnement ${ }^{58}{ }$.

57. Mesures carcérales et mesures non privatives de liberté, Peines de substitution à l'incarcération. Compilation d'outils d'évaluation de la justice pénale, New York, Office des Nations unies contre la drogue et le crime, 2008, p. 1. De plus, Penal Reform International estime que 9 millions de personnes dans le monde sont incarcérées ou détenues dans des conditions qui ne satisfont pas les normes internationales des droits de l'homme applicables et nuisent gravement à leurs chances d'un retour productif dans la société. Le surpeuplement comporte souvent des risques pour la santé publique, compromet la maitrise de la violence à l'intérieur des prisons, crée un environnement dangereux pour le personnel pénitentiaire et rend impossible l'application des normes minimales de détention définies par les Nations unies, qui exigent suffisamment d'éclairage, d'air, de décence et d'intimité " (cité par Dirk van Zyl Smit, op. cit., p. 5).

58. Adalberto Carim Antonio, op. cit., p. 11. 
Quant aux enjeux économiques des peines alternatives, il a été constaté de manière incontestable ${ }^{59}$ que le recours systématique à l'emprisonnement entraîne un coût d'entretien des personnes détenues que l'État a généralement de la peine à supporter. Le coût de l'emprisonnement n'est pas facilement estimable, même à l'échelle mondiale. Cependant, les statistiques de 1997 le situent à environ 62,5 milliards de dollars par an. En général, la masse de prisonniers est très jeune et les investissements pour son entretien sont considérables ${ }^{60}$. Les coûts directs sont généralement constitués de la construction et de l'administration des prisons ainsi que de l'hébergement, de l'alimentation et de la prise en charge sanitaire des détenus ${ }^{61}$.

De plus, le délinquant qui est détenu est également privé de la possibilité d'exercer une activité pourvoyeuse de ressource financière. Cet état de fait le rend non seulement entièrement dépendant du Trésor public, mais surtout prive la société sur la plus-value générée par l'activité que le condamné aurait exercé. Les mesures alternatives qui visent à éviter, au maximum, la privation de liberté devraient permettre de laisser au délinquant sa liberté de travail, de manière à éviter qu'il soit une charge dont l'entretien pèse totalement sur les ressources publiques ${ }^{62}$, et à lui donner la latitude de participer activement à l'effort collectif de construction socioéconomique.

\section{Un régime juridique des peines alternatives favorable à l'amélioration du système de répression}

L'étude du régime juridique des peines alternatives, instituées par le législateur camerounais, devra également permettre de vérifier si elles sont de nature à concourir à une réelle amélioration de son système répressif. Il conviendra dès lors de mesurer l'étendue du champ d'application de ces mesures (2.1) et d'évaluer leurs modalités de mise en œuvre (2.2).

59. Graham Farrell et Ken Clark, What does the world spend on criminal justice?, HEUNI Paper $n^{\circ} 20$, Helsinki, European United Nations Institute for Crime Prevention and Control, 2004.

60. Adalberto Carim Antonio, op. cit.

61. Les prisons sont, en effet, des foyers de maladies telles que la tuberculose et le SIDA, surtout lorsqu'elles sont surpeuplées, et même à la libération des détenus, les risques de propagation sont élevés.

62. À titre d'illustration, le gouvernement malawite, qui a adopté la peine de travail d'intérêt général comme alternative à l'emprisonnement, a économisé en quatre ans (2000-2004) la somme de 227717 dollars (Dirk van Zyl Smit, op. cit., p. 79). 


\subsection{Un champ d'application précis des peines alternatives}

Tel qu'il ressort de la loi $\mathrm{n}^{\mathrm{0}} 2016-007$ du 12 juillet 2016 portant code pénal, le champ d'application matériel des peines alternatives a pour critère de détermination le quantum de la peine encourue par le délinquant. Mais leur champ d'application personnel semble moins nettement déterminable, de même qu'elles peuvent s'appliquer indépendamment de la phase de la procédure concernée ${ }^{63}$.

Relativement aux infractions qui peuvent être sanctionnées par des peines alternatives, les articles 26 (1) et 26-1 (1) disposent qu'aussi bien les travaux d'intérêt général que la sanction-réparation sont « applicables aux délits passibles d'un emprisonnement inférieur à deux ans ou d'une peine d'amende». Ainsi, il convient de relever que la catégorie d'infractions concernée est celle des délits ${ }^{64}$, donc à l'exclusion explicite des crimes qui sont des infractions punies de la peine de mort ou d'une peine privative de liberté dont le maximum est supérieur à dix ans et d'une amende. Ce domaine semble assez restreint, car il ne couvre pas tous les délits. Cette catégorie d'infractions est, par définition, punie d'un emprisonnement d'une durée supérieure à dix jours, mais inférieure à dix ans; la tranche de ces infractions sanctionnées par une peine privative de liberté d'une durée comprise entre deux et dix ans reste, dès lors, exclue du champ d'application des peines alternatives. En adoptant le plafond de deux ans d'emprisonnement comme critère de détermination des infractions susceptibles d'être punies par une peine alternative, le législateur camerounais a pris le contrepied de son homologue français qui, privilégiant la préservation de la liberté du juge, n'a plus entendu limiter l'application des peines alternatives aux seules petites affaires. Ce qui l'a amené à ne fixer aucun plafond au-delà duquel le travail d'intérêt général par exemple ne peut être prononcé ${ }^{65}$. Dans ce dernier système pénal, une peine de travail d'intérêt général, par exemple, ne peut être prononcée, en application de l'article 43-3-1

63. La règle 2.1 des «Règles de Tokyo" prévoit, en effet, que ces règles «s'appliquent à toutes personnes faisant l'objet de poursuites judiciaires, d'un procès ou de l'exécution d'une sentence, à tous les stades de l'administration de la justice pénale ». Le législateur camerounais n'ayant pas fait de spécification, il y a lieu de déduire qu'il s'est conformé à cette exigence.

64. Aux termes de l'art. 21, al. 1 du Code pénal camerounais, "sont qualifiées délits, les infractions punies d'une peine privative de liberté ou d'une peine d'amende lorsque la peine privative de liberté encourue est supérieure à dix (10) jours et n'excède pas dix (10) ans ou que le maximum de l'amende est supérieur à vingt-cinq mille (25000) francs ».

65. Jean Pradel, op. cit., p. 552. 
du Code pénal français, qu'à l'égard de l'auteur d'un délit puni de l'emprisonnement ${ }^{66}$.

De plus, malgré l'apparente clarté des dispositions des articles 26 cidessus, on peut toujours s'interroger sur l'inclusion des contraventions dans ce champ, qui ne vise en principe que «les délits ${ }^{67}$ ». Pourtant, il s'agit des infractions également réprimées par de courtes peines de prison dont les auteurs sont aussi susceptibles d'engorger les prisons. Leur apparente exclusion peut être de nature à constituer un handicap à l'atteinte de l'objectif poursuivi par le législateur. Sauf à admettre que la notion de délit doit s'entendre de manière extensive, comme étant toute infraction, et alors, on pourrait inclure les contraventions dans le domaine des peines alternatives puisqu'elles sont aussi des infractions passibles d'un emprisonnement inférieur à deux ans ou d'une peine d'amende. De plus, les contraventions étant des infractions moins graves que les délits, on pourrait très bien concevoir qu'en vertu du principe selon lequel qui peut le plus peut le moins, le juge devrait être admis à prononcer des peines alternatives lorsque l'infraction en cause est une contravention.

Par ailleurs, le législateur camerounais exclut de manière explicite certaines infractions du champ des peines alternatives. Sans doute, du fait de leur dangerosité pour la société, ou alors, par rapport aux valeurs. Ces exclusions sont contenues à l'article 26-3 du Code pénal et concernent les infractions à la législation sur les armes ${ }^{68}$, les offenses sexuelles $^{69}$ ainsi que les atteintes à l'intégrité corporelle ${ }^{70}$. Alors, même lorsqu'elles rentrent dans la catégorie des délits, ces infractions ne peuvent faire l'objet de peines alternatives.

Cependant, alors que le quantum de la peine d'emprisonnement pouvant permettre le prononcé d'une peine alternative est bien précisé dans son plafond qui est de deux ans, le montant de la peine d'amende n'est pas précisé. On doit donc en déduire qu'il n'y a aucun montant requis à cet effet. Dès lors, quel que soit le montant de la peine d'amende, une peine alternative peut être prononcée par le juge contre l'auteur de l'infraction. Ce critère de délimitation du domaine des peines

66. Voir Cass. crim., Cass. partielle, 15 déc. 1992, dans «Peines - Peine de travail d'intérêt général ", La Semaine juridique. Édition générale, $\mathrm{n}^{\circ}$ 12, 24 mars 1993, 750.

67. En France, dans quelques rares cas, une contravention de $5^{\mathrm{e}}$ catégorie peut être sanctionnée par une peine de travail d'intérêt général. Voir sur ce point Jean Pradel, op. cit., p. 553.

68. Art. 116, 117, 123-1, 237 et 238 du Code pénal camerounais.

69. Art. 268-1, 294, 295 à 298, 346, 347 et 347-1 du Code pénal camerounais.

70. Titre III, chap. 1, art. 275 à 290 du Code pénal camerounais. 
alternatives, retenu par le législateur camerounais, est bien en conformité avec les recommandations de l'ONU contenues dans les "Règles de Tokyo » qui visent «à désengorger les prisons et de permettre d'éviter les courtes peines d'emprisonnement ${ }^{71}$ ».

Quant aux personnes concernées, le législateur camerounais est resté peu précis et laisse déduire que les personnes qui peuvent bénéficier des peines alternatives sont tous les auteurs des infractions punies par l'une de ces peines. D'ailleurs, la règle $\mathrm{n}^{0}$ 5.2.2 des "Règles de Tokyo" recommande expressément cette généralisation lorsqu'elle prévoit que «les présentes Règles s'appliquent sans discrimination de race, de couleur, de sexe, d'âge, de langue, de religion, d'opinion politique ou autre, d'origine nationale ou sociale, de fortune, de naissance ou autre situation ». De plus, l'article 26-3 du Code pénal intitulé «Personnes exclues du bénéfice des peines alternatives » ne retient aussi pour critère de leur détermination que les infractions (infraction à la législation sur les armes, offenses sexuelles et atteintes à l'intégrité corporelle) dont la commission exclut les peines alternatives, sans énoncer véritablement une catégorie de personnes ou même préciser d'autres critères pour les déterminer. Pourtant, compte tenu de l'objectif de resocialisation de certains délinquants, on a émis le souhait que les peines alternatives soient des «stratégies visant à réduire l'emprisonnement dans quatre grands groupes : les enfants, les toxicomanes, les malades mentaux et les femmes, pour lesquels l'emprisonnement a des effets particulièrement préjudiciables». Ces catégories devant pouvoir bénéficier de mesures alternatives à tous les niveaux ${ }^{72}$. En France aussi, le législateur a envisagé au début, dans une loi de 1983, d'exiger au bénéfice de certaines peines alternatives, l'absence d'une condamnation antérieure à plus de quatre mois d'emprisonnement ${ }^{73}$. Elle a finalement abandonné cette condition de telle sorte qu'aujourd'hui, le passé pénal est indifférent en la matière ${ }^{74}$.

71. Exposé des motifs du projet de loi ${ }^{\circ} 989 / \mathrm{PJL} / \mathrm{AN}$ portant Code pénal. Sur les courtes peines d'emprisonnement, voir Muriel Giacopelli, « Approche critique de la courte peine d'emprisonnement", Droit pénal, $\mathrm{n}^{0} 2$, févr. 2014, Lexis 36o, étude 4.

72. Dirk van Zyl Smit, op. cit., p. 61 et suiv.

73. Cass. crim., C., 26 novembre 1986, La Semaine juridique. Édition générale, $\mathrm{n}^{\circ} 7$, 11 février 1987, 100385, qui casse l'arrêt de la Cour d'appel qui condamne un individu pour coups et blessures volontaires ayant entraîné une incapacité totale de travail supérieure à huit jours à l'accomplissement d'un travail d'intérêt général pendant une durée de 240 heures, dans un délai de 18 mois, alors que l'intéressé avait déjà été condamné deux fois pour des délits de droit commun à un an d'emprisonnement.

74. Jean Pradel, op. cit., p. 552. 
Pour les Nations Unies, les peines alternatives sont susceptibles d'être prononcées à tous les stades de la procédure pénale; de la phase de l'enquête de police jusqu'à celle de l'exécution de la peine, en passant par l'instruction préparatoire et le jugement. En ce sens, la règle $\mathrm{n}^{\mathrm{o}} 2.1$ des « Règles de Tokyo » dispose que «les dispositions pertinentes des présentes Règles s'appliquent à toutes personnes faisant l'objet de poursuites judiciaires, d'un procès ou de l'exécution d'une sentence, à tous les stades de l'administration de la justice pénale». Ces règles sont d'ailleurs très génériques, puisqu'elles choisissent le terme "délinquant » comme seule expression pour désigner les personnes susceptibles de prétendre au bénéfice des peines alternatives. Ainsi, il pourra s'agir du suspect, terme par lequel est désignée la personne poursuivie au stade de l'enquête de police, du prévenu lorsqu'elle est en jugement devant le tribunal de première instance connaissant d'un délit, de l'accusé quand elle est encore en jugement devant le tribunal de grande instance siégeant en matière criminelle, ou du condamné lorsque sa culpabilité est établie et que sa peine a été prononcée.

Pour le législateur camerounais cependant, la possibilité de prononcer une peine alternative semble réduite au seul stade du jugement, puisqu'il ressort de l'article 26-1 (2) du Code pénal camerounais que la peine alternative «est prononcée par la juridiction de jugement à la place de l'emprisonnement ou de l'amende, après la déclaration de culpabilité...» En retirant ainsi explicitement la compétence du prononcé d'une peine alternative au parquet ou au juge d'instruction, le législateur camerounais restreint par ce fait même le champ d'application des peines alternatives relativement au moment de la procédure auquel elles peuvent intervenir. Cette restriction doit néanmoins être relativisée, car on trouve par ailleurs d'autres dispositions qui permettent de pallier cette restriction de la loi ${ }^{0}$ 2016-007 du 12 juillet 2016. Il en est ainsi de l'article $246 \mathrm{du}$ Code de procédure pénale, qui permet au juge d'instruction de soumettre l'inculpé à des mesures de surveillance judiciaire ${ }^{75}$ pendant l'instruction préparatoire. De telles mesures, lorsqu'elles sont prises, constituent de véritables mesures alternatives à la détention provisoire qui aurait privé la personne poursuivie de sa liberté en attendant l'issue des opérations d'instruction. Peut-être le législateur de 2016 a procédé à cette restriction, car, s'agissant de peines, seules les juridictions de jugement doivent en avoir la compétence exclusive; mais les mesures privatives de liberté décidées aux autres stades de la procédure pénale

75. Les termes de l'article 246 du Code de procédure pénal camerounais. 
ne produisent-elles pas les mêmes effets nocifs que l'emprisonnement prononcé par le juge de jugement?

\subsection{Des modalités perfectibles de mise en œuvre des peines alternatives}

De prime abord, il sera quelque peu osé de prétendre traiter de modalités de mise en œuvre des peines alternatives telles qu'elles sont prévues par le législateur camerounais du 12 juillet 2016. Lui-même ayant renvoyé leur détermination à des textes particuliers, certainement de nature réglementaire ${ }^{76}$. Elles peuvent cependant être esquissées à partir du contenu des dispositions actuelles relatives à ces sanctions, ou à partir de l'expérience tirée de certains droits étrangers. Certaines de ces modalités sont communes aux deux peines alternatives admises par la loi camerounaise, alors que d'autres sont spécifiques à chacune d'elles.

Relativement aux modalités communes, elles concernent le type d'infraction concernée ainsi que l'exigence pour le juge saisi de prévoir l'emprisonnement encouru en cas d'inexécution de la peine alternative. Il a déjà été relevé que les peines alternatives ne peuvent être prononcées que lorsque l'infraction en cause est un délit passible d'un emprisonnement inférieur à deux ans ou d'une peine d'amende, quel que soit son montant. La présence d'une telle infraction est donc la condition première pour prononcer une peine alternative. Le type de sanction, travail d'intérêt général ou sanction-réparation, est donc indifférent. L'important étant qu'il s'agisse d'un délit qui est surtout puni d'une privation de liberté de courte durée, ou d'une peine pécuniaire dénommée «amende».

L'autre exigence faite au juge qui opte pour une peine alternative est l'obligation de prévoir «la durée de l'emprisonnement encouru en cas d'inexécution de la peine alternative ${ }^{77} »$. Compte tenu du caractère simplement subsidiaire de la peine alternative, la menace de la peine privative de liberté est un moyen de rappeler à l'ordre le délinquant, s'il a tendance à négliger la teneur du bénéfice qui lui est octroyé par l'admission des peines alternatives. Cela rappelle au délinquant que le but du juge est de lui éviter les effets nocifs de l'emprisonnement ou les effets dévastateurs de la peine d'amende sur son patrimoine,

76. Art. 26, al. 6 du Code pénal camerounais dispose que «la liste des travaux d'intérêt général est fixée par un texte particulier». L’art. 26-2 du même Code pénal prévoit de manière générale que «les modalités d'application des peines alternatives sont fixées par un texte particulier».

77. Cette exigence est faite aussi bien pour le prononcé du travail d'intérêt général (C. pén., art. 26, al. 5) que de la sanction réparation (C. pén., art. 26-1, al. 3). 
en préférant la peine alternative. Mais, il doit aussi être conscient que la non-reconnaissance des avantages découlant de cette préférence peut toujours le ramener à la rigueur des peines substituées. Michel Véron ne déclarait-il pas dans ce sens que "qui ne travaille pas, va en prison $^{78}$ »? On estime, en effet, que le condamné à une peine alternative qui se dérobe à ses obligations est traité comme un condamné à une peine d'emprisonnement qui s'évade. Ce qui est assez logique. L'emprisonnement prévu pour être exécuté en cas de non-exécution de la peine alternative peut aussi être assimilé à ce que Philippe Salvage aurait appelé une "peine de la peine» alternative ${ }^{79}$. Cet auteur estime

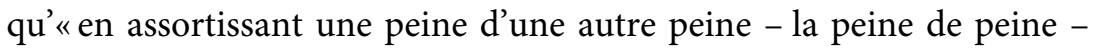
de nature à être exécutée uniquement si la première ne l'est pas, on atteint deux objectifs simultanément: on favorise l'exécution de la première en créant d'emblée une menace à l'égard d'un condamné éventuellement peu coopératif, tout en facilitant, notamment par une procédure simplifiée, la mise en œuvre de la seconde ${ }^{80}$ ».

Cette obligation faite au juge de prévoir la durée de la peine d'emprisonnement à purger en cas d'inexécution de la peine alternative est doublée de l'interdiction qui lui est faite d'assortir cette peine d'emprisonnement de sursis. Jean Pradel estime sur ce point que les peines alternatives constituent déjà une modalité du sursis à l'emprisonnement ${ }^{81}$. La défaillance du délinquant, quant à l'exécution de la peine alternative, déclenche dès lors immédiatement les délais et mécanismes d'exécution de l'emprisonnement substitué. L'admission d'un autre sursis dans ce cas aurait sans doute laissé croire à de la complaisance en faveur du délinquant.

Cependant, il est quelque peu curieux de constater que le législateur ne prévoit que l'emprisonnement comme sanction à l'inexécution par le délinquant de la peine alternative. Il reste muet quant à la peine d'amende qui peut elle aussi avoir été substituée. Est-ce à dire que, dès lors qu'une peine alternative a été prononcée, la peine d'amende qui

78. Michel Véron, "Qui ne travaille pas, va en prison», commentaire sous CA Riom, ch. corr., 16 janv. 2003, A. Nordine: Juris-Data $\mathrm{n}^{0}$ 2003-202796, Droit pénal, $\mathrm{n}^{\circ} 6$, juin 2003 , comm. 73. Pour qui «viole les engagements pris, le condamné qui ne répond pas aux convocations et qui ne fournit pas les pièces exigées pour mettre en œuvre le travail d'intérêt général qu'il a accepté d'accomplir».

79. Philippe Salvage, «Les peines de peine», Droit pénal, $\mathrm{n}^{\circ} 6$, juin 2008, Lexis 360, étude 9 .

80. Ibid.

81. Jean Pradel, op. cit., p. 551. 
aurait été normalement appliquée disparait automatiquement ? Cela traduirait l'instauration d'un régime très souple au bénéfice du délinquant, à travers l'introduction des peines alternatives dans le paysage répressif camerounais. N'aurait-il pas été plus judicieux pour le législateur de prescrire au juge de prévoir aussi bien la durée d'emprisonnement que le montant de l'amende encourue en cas d'inexécution de la peine alternative? Surtout sachant qu'il est fréquent que ces deux sanctions soient conjointement prononcées contre les auteurs de délits. Le législateur camerounais s'est, sur ce point, quelque peu distingué de son homologue français qui, à travers les dispositions de l'article 131-8-1 du Code pénal, prescrit que «lorsqu'elle prononce la peine de sanction-réparation, la juridiction fixe la durée maximum de l'emprisonnement, qui ne peut excéder six mois, ou le montant maximum de l'amende, qui ne peut excéder 15 ooo euros, dont le juge de l'exécution des peines pourra ordonner la mise à exécution en tout ou en partie... ». La peine d'amende ne disparait donc pas, en droit français, du fait du prononcé de la peine alternative qu'est ici la sanction-réparation.

L'une des faiblesses que l'on peut être amené à relever dans la loi camerounaise relative aux peines alternatives est l'absence d'institution d'une autorité chargée de constater l'exécution de la peine alternative à laquelle le délinquant a été condamné. Ailleurs, cette tâche est dévolue au Procureur de la République ${ }^{82}$ ou un magistrat délégué par lui quant à la constatation de l'exécution de la sanction réparation.

Pour ce qui est des modalités particulières à chaque peine alternative, elles concernent soit les bénéficiaires de la sanction, soit l'autorité compétente pour fixer les délais et autres modes d'exécution.

Les bénéficiaires du travail d'intérêt général sont bien précisés par l'article 26, alinéa 1 du Code pénal qui dispose que "cette peine est exécutée en faveur, soit d'une personne morale de droit public, soit d'une personne morale de droit privé chargée d'une mission de service public, soit encore d'un organisme habilité à mettre en œuvre des travaux d'intérêt général ». Le juge devra donc, pour choisir la personne au profit de laquelle un tel travail devra être fait, tenir compte du fait que son activité est exercée pour le bien du public, à l'avantage de tous et qu'elle vise à satisfaire des besoins collectifs, ou tout au moins à un nombre important de personnes dans la société. Le législateur a certainement estimé que l'infraction étant en général un comportement préjudiciable à l'intérêt de la société, du fait du trouble qui est causé contre l'ordre 
initialement établi en faveur de l'ensemble du corps social, c'est à celle-ci, prise dans son ensemble, qu'il convient de réserver le fruit qui pourra découler de l'exécution des condamnations prononcées contre les auteurs de ces infractions. D'ailleurs, le fait pour le Code pénal de prescrire la stricte gratuité ${ }^{83}$ du travail d'intérêt général traduit le souci du législateur de faire de la société le bénéficiaire exclusif de la prestation de travail du condamné. Toute idée de pécule ${ }^{84}$ étant rigoureusement exclue. Par contre, comme dans toutes les législations qui connaissent le travail d'intérêt général ${ }^{85}$, la loi camerounaise conditionne l'application de cette peine au " consentement préalable du prévenu ${ }^{86}$ ». Le législateur a estimé que l'accord du prévenu doit être obligatoirement requis puisque, dans le cas contraire, la prestation accomplie pourrait être assimilée au travail forcé ou obligatoire ${ }^{87}$.

L'idée générale de la fonction réparatrice du droit pénal est donc bien au cœur de l'instauration du travail d'intérêt général comme mesure alternative à l'emprisonnement et à la peine d'amende. Elle l'est d'ailleurs davantage dans l'introduction de la sanction-réparation, sauf que dans ce dernier cas, le bénéficiaire est une personne privée, physique ou morale. Il s'agit en effet d'octroyer une réparation matérielle, selon les termes de

83. C. pén., art. 26, al. 4, d'où il ressort que «le travail d'intérêt général n'est pas rémunéré».

84. Le pécule est entendu comme un «ensemble des sommes inscrites à l'actif d'un détenu dans la comptabilité de l'établissement pénitentiaire où il est incarcéré et comprenant essentiellement la portion de la rémunération qui lui est allouée pour le travail effectué au sein de l'établissement» (Vocabulaire juridique, op. cit., p. 749). En droit camerounais, voir C. pén., art. 25, al. 1 qui prévoit que «Le produit du travail du détenu est affecté ainsi qu'il suit: $-1 / 3$ au Trésor public; $-2 / 3$ à la constitution du pécule». Cette nouvelle répartition faite par le Code pénal est inversement proportionnelle à celle initialement prévue par les dispositions de l'art. 53 du D. n ${ }^{\circ}$ 92-52 du 27 mars 1992, portant régime pénitentiaire au Cameroun, d'où il ressort que « Les sommes résultant des travaux effectués par la main-d'œuvre pénale sont divisées en deux parties: - la première partie représentant les $2 / 3$ des encaissements est perçue par l'agent intermédiaire des recettes et reversée au Trésor public; - la deuxième partie représentant le $1 / 3$ des encaissements est destinée à la constitution d'un pécule qui sera remis au détenu corvéable à la fin de la détention». Ce seront, alors, les dispositions du nouveau Code pénal qui s'appliqueront désormais en la matière.

85. Il en est ainsi de la législation française et de bien d'autres. Voir Françoise Tulkens, «Rapport au symposium, Le centenaire de la peine privative de liberté aux PaysBas », Groningen, 1986, p. 113 et suiv., cité par Jean Pradel, op. cit., p. 152.

86. C. pén., art. 26, al. 2.

87. Règle 3.4 des «Règles de Tokyo» selon laquelle «les mesures non privatives de liberté qui entraînent une obligation pour le délinquant $[. .$.$] , requièrent le$ consentement du délinquant ». 
l'article 26-1 (1) du Code pénal, à celui qui aura individuellement subi, en tant que victime, un préjudice du fait de la commission de l'infraction en cause.

Enfin, la loi $\mathrm{n}^{\mathrm{0}}$ 2016-007 du 12 juillet 2016 donne au juge compétent le pouvoir de fixer le délai dans lequel le condamné à une sanctionréparation doit exécuter une prestation en faveur de la victime, ainsi que les autres modalités de cette exécution. Celles-ci peuvent, par exemple, être relatives à la détermination de la prestation matérielle à exécuter au bénéfice de la victime, ou au prononcé d'une astreinte qui viserait à inciter le débiteur de la prestation à se hâter d'exécuter la réparation. Ce qui n'est pas le cas pour les travaux d'intérêt général dont la liste ${ }^{88}$, les conditions et modalités d'exécution ne peuvent être déterminées que par un texte réglementaire. Les pouvoirs du juge semblent alors, sur cette sanction, confinés à la simple option de prononcer ou non la peine de travail d'intérêt général et cela seulement entre le plancher de 200 heures et le plafond de 240 heures prédéterminées par le Code pénal ${ }^{89}$. En déterminant cette fourchette dans le texte de loi, le législateur camerounais n'a pas entendu laisser la liberté au juge pour procéder à la fixation de la durée du travail d'intérêt général auquel il pouvait condamner le délinquant.

En définitive, les questions liées aux effets nocifs des peines privatives de liberté se posent au Cameroun avec la même acuité que dans beaucoup d'autres pays. En choisissant de se conformer aux «Règles de Tokyo " pour intégrer dans le droit pénal camerounais les peines alternatives, le législateur du 12 juillet 2016 a entendu apporter des solutions adéquates au problème de la surpopulation carcérale et ses corolaires, que sont les risques d'atteintes aux droits fondamentaux des détenus ou d'échec de l'objectif de resocialisation du délinquant. Cependant, si l'opportunité de l'introduction de ces mesures alternatives est évidente, le succès quant à l'atteinte des objectifs poursuivis sera inévitablement tributaire des modalités de mise en œuvre desdites mesures. Les pouvoirs publics devraient dès lors prendre la mesure des enjeux de l'introduction des peines alternatives au Cameroun en vue d'élaborer ces modalités, encore attendues, en toute conséquence.

Maître de conférence à la faculté des Sciences juridiques et politiques Université de Dschang (Cameroun)

88. C. pén., art. 26, al. 6 qui dispose que «la liste des travaux d'intérêt général est fixée par un texte particulier».

89. C. pén., art. 26 , al. 3 . 